\title{
Phorbol Ester and A23187 Have Additive but Mechanistically Separate Effects on Vasopressin Action in Rabbit Collecting Tubule
}

\author{
Yasuhiro Ando, Harry R. Jacobson, and Matthew D. Breyer \\ Division of Nephrology, Department of Medicine, Vanderbilt University, Nashville Tennessee 37232; \\ and Veterans Administration Medical Center, Nashville, Tennessee 37212
}

\begin{abstract}
Activation of protein kinase C (PKC) and elevation of intracellular calcium ion concentration $\left(\left[\mathrm{Ca}^{++}\right]_{\mathrm{i}}\right)$ result from phosphatidylinositol biphosphate (PIP $)$ breakdown. We previously demonstrated that PKC activation inhibits arginine vasopressin (AVP)-induced osmotic water flow in rabbit cortical collecting tubule $(\mathrm{CCT})$ perfused in vitro at $37^{\circ} \mathrm{C}$. To estimate the potential significance of $\mathrm{PIP}_{2}$ turnover as a modulator of water transport in this nephron segment, we examined the effect of Ca on AVP action and explored the mechanisms of action of PKC and increased $\left[\mathrm{Ca}^{++}\right]_{i}$.

In rabbit CCTs perfused at $37^{\circ} \mathrm{C}$, pretreatment with bath A23187 $\left(2 \times 10^{-8} \mathrm{M}, 2 \times 10^{-6} \mathrm{M}\right)$, a Ca ionophore, almost totally suppressed AVP $(10 \mu \mathrm{U} / \mathrm{ml})$-induced peak hydraulic conductivity (Lp). The suppression by $2 \times 10^{-8} \mathrm{M}$ A23187 was as potent as that by $2 \times 10^{-6} \mathrm{M} \mathbf{A 2 3 1 8 7}$, and significant even when it was administered $10 \mathrm{~min}$ after AVP. When phorbol myristate acetate (PMA, $1^{-9} \mathrm{M}$ ), a PKC activator, and A23187 $\left(2 \times 10^{-8} \mathrm{M}\right)$ were placed in the bath simultaneously, the combined suppressive effect on peak $L p$ was greater than that of either inhibitor alone. However, the mechanisms of inhibition by PMA and A23187 were different. While both $10^{-7}$ and $10^{-9} \mathrm{M}$ PMA suppression are primarily post-cAMP, A23187 predominantly suppressed a pre-cAMP step: $10^{-4} \mathrm{M}$ chlorophenylthio-cAMP-induced peak $L p$ was not affected by $2 \times 10^{-8} \mathrm{M} \mathrm{A23187}$, and only partially inhibited by $2 \times 10^{-6}$ M A23187. The PMA $\left(1^{-7} \mathrm{M}\right)$ suppression of AVP-induced peak Lp was totally reversed by bath staurosporine $\left(10^{-7} \mathrm{M}\right)$, a PKC inhibitor, but not attenuated by either bath indomethacin $\left(5 \times 10^{-6} \mathrm{M}\right)$ or low Ca $\left(1-2 \times 10^{-6} \mathrm{M}\right)$ bath medium. In contrast, the $A 23187\left(2 \times 10^{-8} \mathrm{M}\right)$ suppression of the peak $L_{p}$ was not affected by staurosporine, but was significantly reversed by indomethacin or low $\mathrm{Ca}$ bath medium.

We conclude: (a) Elevation of $\left[\mathrm{Ca}^{++}\right]_{i}$, as well as activation of PKC, suppresses the hydroosmotic effect of AVP.on CCT at $37^{\circ} \mathrm{C}$. (b) When stimulated simultaneously these two intracellular mediators are additive in their antagonism of AVP action. These results suggest that stimulated PIP $_{2}$ breakdown may be an important modulator of water transport in CCT. (c) Differ-
\end{abstract}

Parts of this study were presented at the 10th International Congress of Nephrology, 1987, in London, and at the Annual Meeting of the American Society of Nephrology, 1987, in Washington, D.C.

Address reprint requests to Dr. Breyer, Div. of Nephrology, Vanderbilt University. 1987.

Received for publication 7 July 1987 and in revised form 16 October

J. Clin. Invest.

(c) The American Society for Clinical Investigation, Inc.

0021-9738/88/05/1578/07 \$2.00

Volume 81, May 1988, 1578-1584 ent mechanisms underlie PKC and Ca-mediated suppression of the AVP-induced water transport. The inhibition of AVP action by increased $\left[\mathrm{Ca}^{++}\right]_{\text {is }}$ is primarily pre-cAMP, and involves a cyclooxygenase metabolite(s) of arachidonic acid, while the inhibition by PKC is post-cAMP, and independent of cyclooxygenase products of arachidonic acid.

\section{Introduction}

Activation of protein kinase $\mathrm{C}(\mathrm{PKC})^{1}$ and elevation of intracellular calcium ion concentration $\left(\left[\mathrm{Ca}^{++}\right]_{\mathrm{i}}\right)$ are two major signal transduction pathways employed by many biologically active agents and hormones which stimulate phosphatidylinositol biphosphate (PIP $)$ breakdown (1-3). In many types of mammalian cells, both pathways are known to act synergistically $(1,3,4)$. While we have demonstrated that PKC activators markedly inhibit arginine vasopressin (AVP)-induced osmotic water flux in rabbit cortical collecting tubule (CCT) (5), studies addressing the role for increased $\left[\mathrm{Ca}^{++}\right]_{\mathrm{i}}$ in modulating AVP action have yielded conflicting results. Goldfarb (6) showed that increased bath $\mathrm{Ca}$ concentration augmented AVP-induced water transport in rabbit CCT at $37^{\circ} \mathrm{C}$, although Jones et al. (7) failed to reproduce such an effect. Dillingham et al. (8) showed a stimulatory effect of the Ca ionophore A23187 on AVP-induced osmotic water flux in rabbit CCT at $25^{\circ} \mathrm{C}$. Conversely, Jones et al. (9) conducted similar experiments at $37^{\circ} \mathrm{C}$ using another $\mathrm{Ca}$ ionophore, ionomycin, and found that it suppressed AVP action.

In evaluating the PIP $_{2}$ breakdown cascade as a potential modulator of AVP action in CCT, it is important to clarify the role of the Ca-mediated pathway as well as that of the PKC pathway, since both are activated simultaneously. Therefore we investigated the role for $\left[\mathrm{Ca}^{++}\right]_{\mathrm{i}}$ by using the $\mathrm{Ca}$ ionophore A23187. In addition, we examined the combined effect of PMA and A23187 to look for the interaction between these two pathways. Finally, we explored and compared possible mechanisms of action for PKC and increased $\left[\mathrm{Ca}^{++}\right]_{i}$.

\section{Methods}

Single CCTs (1.0-2.5 mm in length) were dissected from kidneys of female New Zealand white rabbits weighing $1.5-2.0 \mathrm{~kg}$ and perfused using methods described previously (5). The flow rate of bath solution was adjusted to $0.5 \mathrm{ml} / \mathrm{min}$. The collection rate of perfusate was adjusted to $15-20 \mathrm{nl} / \mathrm{min}$. The perfused fluid was collected in a constriction pipette of known volume (between 65 and $150 \mathrm{nl}$ ).

The composition of standard bath medium, dissection medium, and isotonic perfusate were as follows (in millimolars): $\mathrm{NaCl}, 105$;

1. Abbreviations used in this paper: $\left[\mathrm{Ca}^{++}\right]_{\mathrm{i}}$, intracellular calcium ion concentration; CcAMP, chlorophenylthio-cyclic AMP; CCT, cortical collecting tubule; IND, indomethacin; Lp, hydraulic conductivity; $\mathrm{PIP}_{2}$, phosphatidylinositol biphosphate; PKC, protein kinase C; SSP, staurosporine. 
$\mathrm{NaHCO}_{3}, 25 ; \mathrm{Na}$ acetate, $10 ; \mathrm{NaHPO}_{4}, 2.3 ; \mathrm{KCl}, 5 ; \mathrm{CaCl}_{2}, 1.8$ $\mathrm{MgSO}_{4}, 1.0$; glucose, 8.3; and alanine, 5 (osmolality 300 mosmol). Low $\mathrm{Ca}$ bath medium contained no $\mathrm{CaCl}_{2}$, but the other components were the same as standard bath medium. Measured $\mathrm{Ca}$ concentration of low $\mathrm{Ca}$ bath medium was between 0.001 and $0.002 \mathrm{mM}$. The composition of hypotonic perfusate was identical to that of isotonic perfusate except for the low osmolality ( 150 mosmol), due to lower $\mathrm{NaCl}$ concentration $(30 \mathrm{mM})$, and the addition of tritium-labeled inulin as a volume marker. Both isotonic and hypotonic perfusate contained 0.2 $\mathrm{mg} / \mathrm{ml}$ Food, Drug and Cosmetic dye No. 3 (Aniline and Chemical Co., Chicago, IL) to detect cell damage and perfusate leak (5). Before use, all solutions were bubbled to equilibration at $37^{\circ} \mathrm{C}$ with a $95 \%$ $\mathrm{O}_{2} / 5 \% \mathrm{CO}_{2}$ gas mixture to achieve a pH of $\sim 7.40$ and a $\mathrm{pCO}_{2}$ of $\sim 40$ torr. Bath medium was held in a silicon-coated glass syringe mounted on a infusion pump (Sage Instruments, Inc., Cambridge, MA). Hydraulic conductivity ( $\mathrm{Lp}$, centimeters per atmosphere [atm] per second) was calculated according to DuBois et al. (10):

$(1 / R T S) \cdot(1 / O b)^{2} \cdot[O b \cdot(V i-V o)+O i \cdot V i \cdot \operatorname{Ln}\{(O b-O i) \cdot V i\}$

$(\mathrm{Ob} \cdot \mathrm{Vo}-\mathrm{Oi} \cdot \mathrm{Vi})\}$ ],

where $\mathbf{R}$ is the gas constant, $\mathbf{T}$ is temperature of bath medium (degree kelvin), $S$ is luminal surface area of the tubule calculated from tubule length as measured directly by eyepiece reticle at the end of each experiment and an assumed lumen diameter of $20 \mu \mathrm{m}, \mathrm{Ob}$ and $\mathrm{Oi}$ are osmolality of bath medium and perfusate, $\mathrm{Vo}$ and $\mathrm{Vi}$ are collection and perfusion rate $(\mathrm{Vo}=\mathrm{Vp} / \mathrm{t}, \mathrm{Vi}=\mathrm{Vo} \cdot \mathrm{Co} / \mathrm{Ci}: \mathrm{Vp}$; volume of constriction pipette, $\mathrm{t}$; collection time, $\mathrm{Co}$ and $\mathrm{Ci}$; and radioactivity [counts per minute per nanoliter] of collected fluid and perfusate. Co and $\mathrm{Ci}$ were counted by a liquid scintillation counter ([model 6892; Tracor Analytic Co., Des Plaines, IL]).

\section{Experimental protocol at $25^{\circ} \mathrm{C}$ (Fig. 1)}

These experiments were conducted to confirm the finding of Dillingham et al. (8) that the $\mathrm{Ca}$ ionophore A23187 has a stimulatory

\section{EXPERIMENT AT $25^{\circ} \mathrm{C}$}

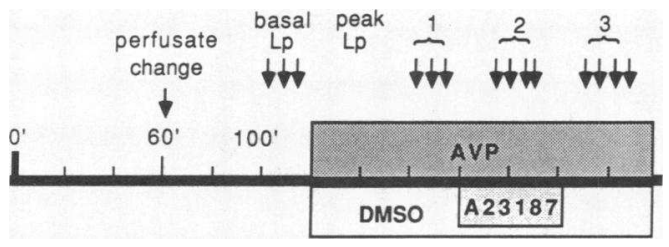

EXPERIMENTS AT $37^{\circ} \mathrm{C}$

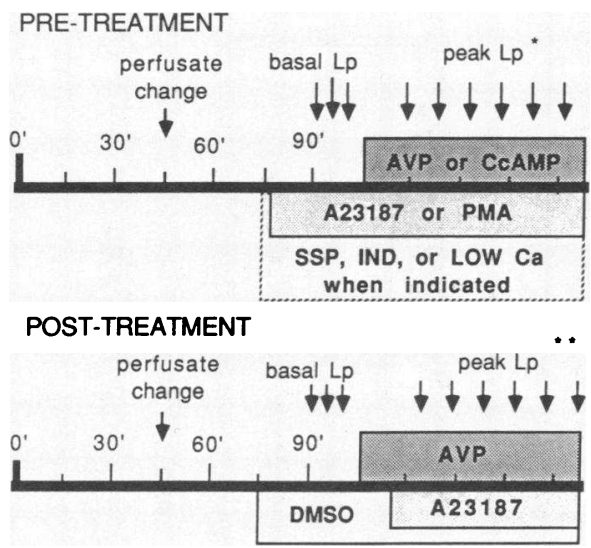

Figure 1. Summary of experimental proticols. See text for details and abbreviations. *Peak Lp was calculated as mean of the three largest values during exposure to AVP. **Peak Lp was calculated as mean of the three largest values during 30 to $50 \mathrm{~min}$ after the addition of AVP. effect on AVP-induced peak Lp at this temperature. Therefore the same concentrations of AVP and A23187 used in their studies were chosen. Pure DMSO was used as the solvent for A23187. The final concentration of DMSO in bath medium during AVP exposure was $0.01 \%$ by volume. We have already shown that this concentration of DMSO has no effect on basal or AVP-induced peak Lp (5). During the first $60 \mathrm{~min}$ of incubation, tubules were perfused with isotonic perfusate. Subsequently the perfusate was changed to hypotonic perfusate. After an additional $45 \mathrm{~min}$, three collections were made to determine basal Lp. Then $50 \mu \mathrm{U} / \mathrm{ml}$ AVP was added to bath medium. $40 \mathrm{~min}$ after addition of AVP, three collections were made for determination of peak Lp. Then $10^{-7} \mathrm{M}$ A23187 was added to bath medium. 15-20 min after addition of A23187, three to four collections were made to see the effect of ionophore on peak Lp. Subsequently A23187 was removed from the bath and three to four recovery collections were made 10-30 min after the removal of A23187. In each period, the peak Lp was calculated as the mean of all collections.

\section{Experimental protocols at $37^{\circ} \mathrm{C}$ (Fig. 1)}

To compare the data with our previous studies (5), each experiment was purposely conducted on an identical time schedule. During the first 30-45 min of incubation in all experiments, tubules were perfused with isotonic perfusate. Subsequently the perfusate was changed to hypotonic perfusate. In control studies, after $90 \mathrm{~min}$ of incubation three collections were made for calculation of basal Lp, then $10 \mu \mathrm{U} / \mathrm{ml}$ AVP was added to the bath and six to nine collections were made during the next 15-50 min to determine peak Lp. In DMSO control studies, $0.01 \%$ DMSO was added to bath medium $15 \mathrm{~min}$ before basal collections. This time schedule for basal and peak collections was also kept in all following experimental protocols except for protocol 2. A submaximal AVP dose was chosen theoretically to enable observation of either suppressive or stimulatory effects of the agents examined. Both in control and experimental studies, the peak Lp was defined as the mean of the three largest values during exposure to AVP. Peak Lp was usually observed $25-45$ min after the addition of AVP.

1. Effect of A23187 on AVP response. This ionophore $\mathrm{A} 23187$ was added to bath medium 10-15 min before the basal collections to increase $\left[\mathrm{Ca}^{++}\right]_{i}(3,4,11)$. AVP was then added and peak $L p$ was determined as defined above.

2. Effect of $A 23187$ posttreatment on $A V P$ response. This protocol served to determine whether $\left[\mathrm{Ca}^{++}\right]_{\mathrm{i}}$ increased after initiation of the AVP response can affect AVP-induced water movement. In these studies, $0.01 \%$ DMSO was added to bath medium $15 \mathrm{~min}$ before the basal collections. Then, $10 \mathrm{~min}$ after exposure to $10 \mu \mathrm{U} / \mathrm{ml}$ AVP, A23187 was added to bath medium. Collections were started soon after the addition of A23187 and six to nine collections were made. Peak Lp of both control and experimental studies were calculated as the mean of the three largest values during 30-50 min after the addition of AVP.

3. Combined effect of phorbol myristate acetate (PMA) and A23187. These experiments were designed to examine the interaction between PKC and increased $\left[\mathrm{Ca}^{++}\right]_{\mathrm{i}}$ which synergize in many other systems $(3,4,12)$. PMA and A23187 were simultaneously added to the bath in the same experimental program as protocol 1.

4. Effect of A23187 on post-cAMP events. $10^{-4} \mathrm{M}$ chlorophenylthio-cyclic AMP (CcAMP) was applied instead of AVP with or without A23187 pretreatment.

5. Effect of staurosporine (SSP) on PMA action. SSP is a potent PKC inhibitor recently described (13). Experiments were designed to see how SSP affects the action of PMA and A23187. $10^{-7}$ M SSP was added to bath medium 15 min before basal collections. PMA or A23187 was added to the bath $5 \mathrm{~min}$ after SSP.

6. Effect of indomethacin (IND) on the actions of PMA and $A 23187$. This protocol was designed to see if cyclooxygenase metabolites of arachidonate mediate the actions of these compounds. $5 \times 10^{-6}$ $M$ IND was chosen because this concentration is known to inhibit cyclooxygenase almost completely but have minimal suppressive effect on cAMP phosphodiesterase (14). IND, PMA, and A23187 were added to the bath in the same way as in protocol 5 . 


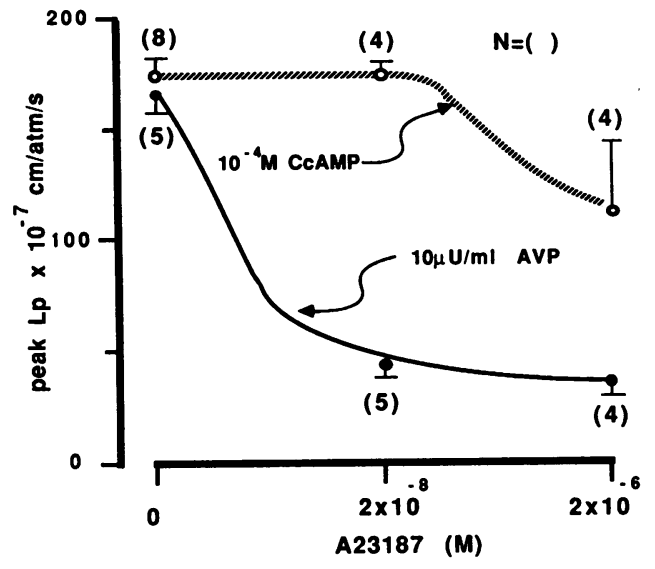

Figure 2. Effects of A23187 on AVP or CcAMP-induced peak Lp. Circles and bars represent means and SE, respectively. $N$, Number of experiments. At $2 \times 10^{-8} \mathrm{M}, \mathrm{A} 23187$ produced almost maximal suppression of the AVP response, but had no effect on the CcAMP response.

7. Effect of low bath Ca on the actions of PMA and A23187. To test whether the effect of either of these agents is dependent on peritubular $\mathrm{Ca}$, bath medium was changed to low $\mathrm{Ca}$ medium 15 min before basal collections. PMA or A23187 was administered 5 min after the bath $\mathrm{Ca}$ was reduced.

In all studies using PMA, the syringe holding the bath medium was wrapped with aluminum foil to prevent degeneration of PMA by light. Pure DMSO was used as the solvent for A23187, PMA, and SSP. The final concentration of DMSO in bath medium in all experiments using A23187, PMA, or SSP was $0.01 \%$ by volume.

\section{Reagents}

A23187, AVP, CcAMP, DMSO, IND, and PMA were purchased from Sigma Chemical Co., St. Louis, MO. Tritium-labeled inulin was purchased from E. I. DuPont, NEN Products, Boston, MA. AVP, CcAMP, DMSO, and PMA used in this study were aliquots of the same stock as used in our previous study (5). SSP (Kyowa Hakko Kogyo, Tokyo, Japan) was a generous gift from Dr. Seymour Mong.

\section{Statistics}

Data are presented as mean \pm SE, and statistical analyses were made by using nonpaired $t$ test unless indicated. Differences with $P<0.05$ were considered statistically significant.

\section{Results}

Experiments at $25^{\circ} \mathrm{C}$

In five experiments, $50 \mu \mathrm{U} / \mathrm{ml}$ AVP increased $L p\left(\times 10^{-7} \mathrm{~cm} /\right.$ atm per s) from a basal of $3.5 \pm 0.3$ to a peak of $90.1 \pm 7.5$. Superimposed A23187 $\left(10^{-7} \mathrm{M}\right)$ further increased the peak $\mathrm{Lp}$ up to $124.8 \pm 5.6(P<0.01)$. After removal of A23187, peak Lp decreased to $106.4 \pm 4.0 \times 10^{-7} \mathrm{~cm} / \mathrm{atm}$ per $\mathrm{s}$, though this change was not statistically significant. These results were essentially the same as those shown by Dillingham et al. (8).

\section{Experiments at $37^{\circ} \mathrm{C}$}

In control studies, $10 \mu \mathrm{U} / \mathrm{ml}$ AVP increased $\mathrm{Lp}\left(\times 10^{-7} \mathrm{~cm} /\right.$ atm per s) from a basal of $5.0 \pm 0.6$ to a peak of $166.0 \pm 5.9(n$ $=15$ ). Both of these values were identical to those of the DMSO control group (basal, 4.7 \pm 1.4 ; peak, $166 \pm 10.4 ; n=5$ ).

1. Effect of A23187 on AVP response. Two different doses of A23187 $\left(2 \times 10^{-8}\right.$ and $\left.2 \times 10^{-6} \mathrm{M}\right)$ were examined $(n=5$ and 4 , respectively). The basal $\mathrm{Lp}\left(\times 10^{-7} \mathrm{~cm} / \mathrm{atm}\right.$ per s) was slightly increased by $2 \times 10^{-8}$ or $2 \times 10^{-6} \mathrm{M} \mathrm{A23187}$ pretreatment $(12.8 \pm 2.0$ and $12.6 \pm 1.7$, respectively; $P<0.01$ vs. DMSO control basal $\mathrm{Lp}$ in either case). However, in contrast to the result at $25^{\circ} \mathrm{C}$, AVP-induced peak Lp was markedly suppressed by A23187 as shown in Fig. 3. The low dose of A23187 was as potent as the high dose: AVP-induced peak Lp $\left(\times 10^{-7} \mathrm{~cm} / \mathrm{atm}\right.$ per s) was $44.8 \pm 4.4$ with $2 \times 10^{-8} \mathrm{M}$ A23187 pretreatment and $37.6 \pm 4.4$ with $2 \times 10^{-6} \mathrm{M}$ A23187 pretreatment, respectively $(P<0.001$ vs. DMSO control peak Lp in either case). This suggests that $2 \times 10^{-8} \mathrm{M}$ is close to the maximal dose for A23187 suppression of the AVP-induced peak Lp in this system. Thus to avoid nonspecific or toxic effects of A23187 $(11,12)$ discussed later, the low dose (2 $\times 10^{-8} \mathrm{M}$ ) was applied in the following studies with the exception of protocol 4.

2. Effect of $A 23187$ posttreatment on $A V P$ response. In five experiments, basal $\mathrm{Lp}\left(3.03 \pm 0.7 \times 10^{-7} \mathrm{~cm} / \mathrm{atm}\right.$ per s) was not different from that of DMSO control. Like PMA posttreatment (5), the addition of $2 \times 10^{-8} \mathrm{M} \mathrm{A23187} 10 \mathrm{~min}$ after 10 $\mu \mathrm{U} / \mathrm{ml}$ AVP suppressed the peak $\mathrm{Lp}\left(\times 10^{-7} \mathrm{~cm} / \mathrm{atm}\right.$ per $\left.\mathrm{s}\right)$ significantly to $99.2 \pm 11.0(n=5)$ compared with the DMSO control peak $\operatorname{Lp}(165.1 \pm 10.0, n=5, P<0.01)$.

3. Combined effect of PMA and A23187 (Fig. 3). For this study we chose a submaximal dose of PMA $\left(10^{-9} \mathrm{M}\right)(5)$ with a near maximal dose of A23187 $\left(2 \times 10^{-8} \mathrm{M}\right)$. The maximal dose $\left(10^{-7} \mathrm{M}\right)$ of PMA is inadequate to use in this protocol, since in our previous study (5) it completely abolished the increase in Lp after AVP administration. In four experiments, by pretreating with these agents simultaneously, the basal $L p$ $\left(\times 10^{-7} \mathrm{~cm} / \mathrm{atm}\right.$ per s) was increased to $18.4 \pm 2.6(P<0.001 \mathrm{vs}$. DMSO control). This value was higher, though not significantly, than that of $10^{-9} \mathrm{M}$ PMA $\left(5.9 \pm 2.9 \times 10^{-7} \mathrm{~cm} / \mathrm{atm}\right.$ per s) or $2 \times 10^{-8} \mathrm{M}$ A23187 alone. The peak Lp after AVP

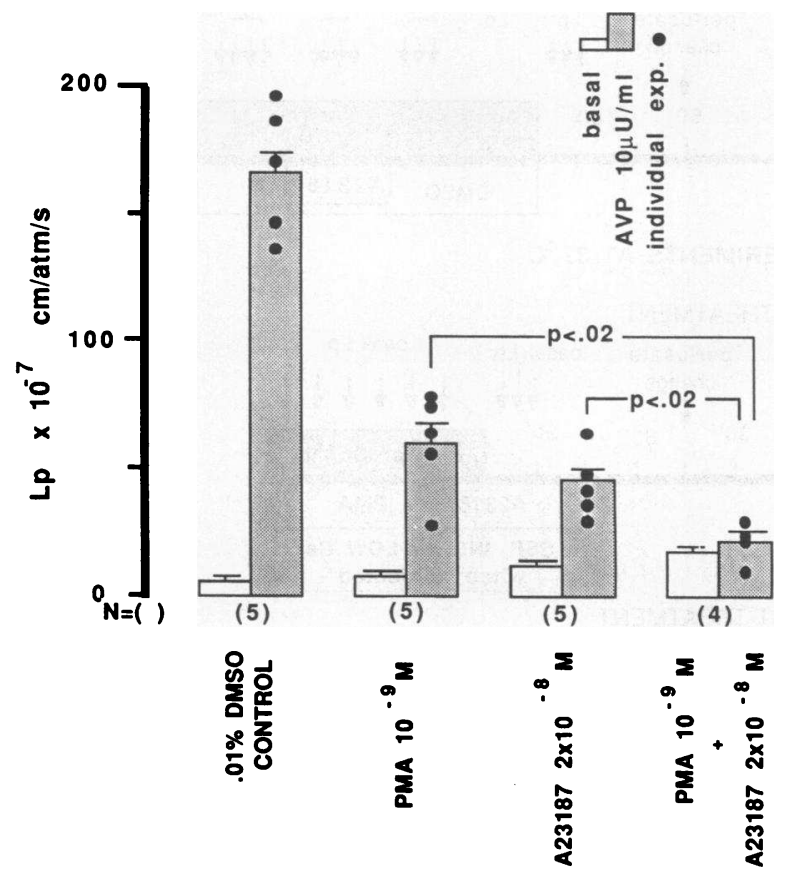

Figure 3. Effect of combined administration of PMA and A23187 on the AVP-induced peak Lp. Bars above each column represent SE. N, Number of experiments. These two agents are additive in their suppression of the AVP response. 
addition was almost completely suppressed $\left(24.0 \pm 3.5 \times 10^{-7}\right.$ $\mathrm{cm} / \mathrm{atm}$ per $\mathrm{s})$. This suppression of peak $\mathrm{Lp}\left(\times 10^{-7} \mathrm{~cm} / \mathrm{atm}\right.$ per s) was significantly greater than that by $10^{-9}$ M PMA $(59.3 \pm 6.0, n=5)$ or $2 \times 10^{-8} \mathrm{M} \mathrm{A23187}(44.8 \pm 4.4, n=5)$ alone $(P<0.02$ in both cases).

4. Effect of $A 23187$ on post-cAMP events. In eight control studies, $10^{-4} \mathrm{M}$ CcAMP increased $\mathrm{Lp}\left(\times 10^{-7} \mathrm{~cm} /\right.$ atm per s) from a basal of $4.2 \pm 0.7$ to a peak of $177.9 \pm 14$.1. Basal $\mathrm{Lp}$ $\left(\times 10^{-7} \mathrm{~cm} / \mathrm{atm}\right.$ per s) was increased by either dose of A23187 (15.5 \pm 2.3 with $2 \times 10^{-8} \mathrm{M}$, and $16.6 \pm 1.9$ with $2 \times 10^{-6} \mathrm{M}$, respectively; $P<0.01$ vs. control basal Lp in both cases). As shown in Fig. 3, the CcAMP-induced peak $\mathrm{Lp}\left(\times 10^{-7} \mathrm{~cm} / \mathrm{atm}\right.$ per s) was not suppressed by $2 \times 10^{-8} \mathrm{M} \mathrm{A23187}(177.1 \pm 5.0, n$ $=4$ ), and was only partially suppressed by $2 \times 10^{-6} \mathrm{M}$ A23187 (110.5 $\pm 17.7, P<0.05$ vs. control).

5. Effect of SSP on the action of PMA and A23187 (Fig. 4). In four SSP control experiments, $10^{-7} \mathrm{M}$ SSP pretreatment itself affected neither the basal $\operatorname{Lp}\left(4.4 \pm 0.9 \times 10^{-7} \mathrm{~cm} / \mathrm{atm}\right.$ per s) nor the AVP-induced peak $\operatorname{Lp}\left(172.1 \pm 11.6 \times 10^{-7} \mathrm{~cm} / \mathrm{atm}\right.$ per s). However, the suppression of peak Lp by $10^{-7} \mathrm{M}$ PMA was totally reversed by SSP; the PMA pretreated peak Lp in the presence of SSP $\left(165.2 \pm 7.5 \times 10^{-7} \mathrm{~cm} /\right.$ atm per $\left.\mathrm{s}, n=4\right)$ was statistically the same as the SSP control peak Lp. Conversely the suppression by $2 \times 10^{-8} \mathrm{M}$ A23187 was not significantly attenuated by SSP. The peak Lp with SSP and A23187 pretreatment was $53.4 \pm 6.1 \times 10^{-7} \mathrm{~cm} /$ atm per $\mathrm{s}(n=4)$.

6. Effect of IND on the actions of PMA and A23187 (Fig. 5). In four IND control experiments, $5 \times 10^{-6} \mathrm{M}$ IND pretreatment itself had no effect on basal or peak $\mathrm{Lp}(6.9 \pm 2.7$ and $166.4 \pm 20.5 \times 10^{-7} \mathrm{~cm} /$ atm per s, respectively). $10^{-7} \mathrm{M}$ PMA completely suppressed the AVP effect on Lp despite the presence of IND: basal and peak Lp were 10.9 \pm 2.1 and 14.6 \pm 2.0 $\times 10^{-7} \mathrm{~cm} / \mathrm{atm}$ per $\mathrm{s}$, respectively ( $n=4$, not significant by paired $t$ test). IND also failed to attenuate the suppression by a submaximal dose of PMA: the PMA $\left(10^{-9} \mathrm{M}\right)$ pretreated peak Lp in the presence of IND $\left(52.2 \pm 7.1 \times 10^{-7} \mathrm{~cm} / \mathrm{atm}\right.$ per $\mathrm{s}, n$ $=4$ ) was not significantly different from that without IND. In marked contrast, IND significantly attenuated the suppression

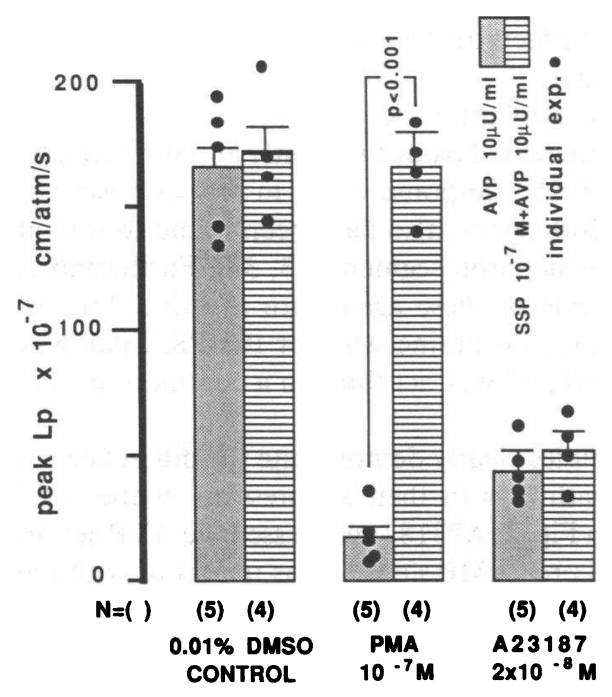

Figure 4. Reversal effect of SSP on the peak Lp suppression by PMA or A23187. Bars above each column represent SE. N, Number of experiments. SSP totally reversed the PMA suppression of AVP-induced peak Lp, but had no effect on the A23187 suppression.

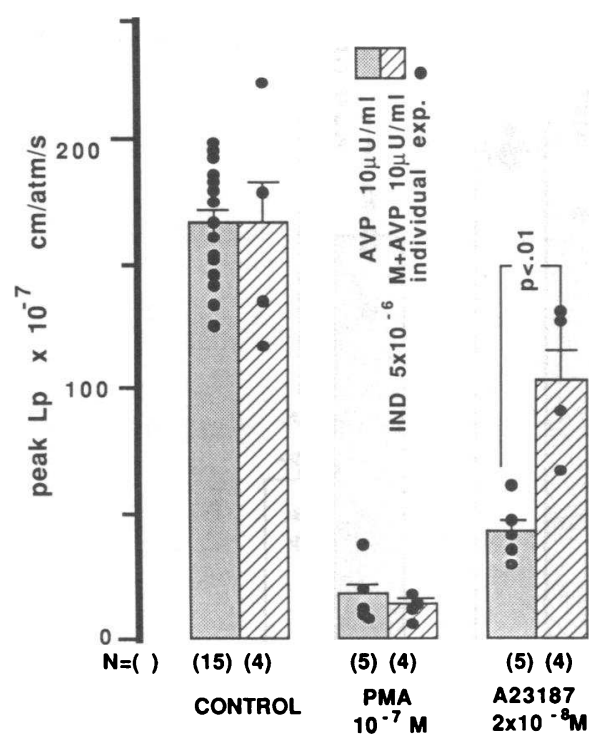

Figure 5. Effect of IND on the peak Lp suppression by PMA or A23187. Bars above each column represent SE. N, Number of experiments. IND had no effect on the PMA suppression but significantly attenuated the $\mathrm{A} 23187$ suppression.

of peak $\mathrm{Lp}\left(\times 10^{-7} \mathrm{~cm} / \mathrm{atm}\right.$ per s) by $2 \times 10^{-8} \mathrm{M} \mathrm{A23187}$ (105.0 \pm 13.4 with IND, $n=4$, and $44.8 \pm 4.4$ without IND, $n$ $=5, P<0.01)$.

7. Effect of low bath Ca on the actions of PMA and $A 23187$. In six low $\mathrm{Ca}$ bath control experiments, low peritubular $\mathrm{Ca}$ concentration had no effect on basal $\mathrm{Lp}(6.2 \pm 1.0$ $\times 10^{-7} \mathrm{~cm} / \mathrm{atm}$ per $\mathrm{s}$, not significant vs. control basal Lp with normal bath medium), but augmented the AVP-induced peak Lp response $\left(223.6 \pm 14.5 \times 10^{-7} \mathrm{~cm} /\right.$ atm per $\mathrm{s}, P<0.001$ vs. control peak Lp with normal bath medium). Even under this condition, $10^{-7} \mathrm{M}$ PMA still suppressed AVP response completely: the basal and the peak $L p$ was $28.8 \pm 2.5$ and $33.0 \pm 1.8$ $\times 10^{-7} \mathrm{~cm} /$ atm per s, respectively ( $n=4$, not significant by paired $t$ test). Conversely, the suppression of the peak Lp by 2 $\times 10^{-8}$ M A23187 was attenuated $\left(138.0 \pm 7.9 \times 10^{-7} \mathrm{~cm} / \mathrm{atm}\right.$ per $\mathrm{s}, n=4)$. Since lowering bath $\mathrm{Ca}$ concentration itself significantly changed the peak Lp response to AVP, the effect of low Ca bath on the PMA or A23187 suppression was estimated by calculating percent inhibition of peak Lp. As shown in Fig. 6, while the degree of inhibition by $10^{-7}$ M PMA was unaffected by low $\mathrm{Ca}$ bath $(88.8 \pm 2.9 \%$ with normal bath and $85.2 \pm 0.9 \%$ with low $\mathrm{Ca}$ bath), that by $2 \times 10^{-8} \mathrm{M} \mathrm{A23187}$ was significantly attenuated $(73.0 \pm 2.6 \%$ in normal bath and $38.3 \pm 3.5 \%$ in low Ca bath, $P<0.001$ ).

\section{Discussion}

Our knowledge about the role of $\mathrm{PIP}_{2}$ breakdown in signal transduction is expanding rapidly. The individual roles of PKC and increased $\left[\mathrm{Ca}^{++}\right]_{\mathrm{i}}$ and the complex interactions between these two branches of PIP $_{2}$ breakdown cascade in regulating cell function are being elucidated in various mammalian tissues $(1-4,15,16)$. These intracellular mediators also have been found to interact with the other signal transduction pathways, especially the cAMP-mediated pathway $(3,4)$. The type of interaction between the $\mathrm{PIP}_{2}$ breakdown-initiated pathways and the CAMP-mediated pathway differs from tissue to tissue. These signalling pathways can be additive or antagonistic in 


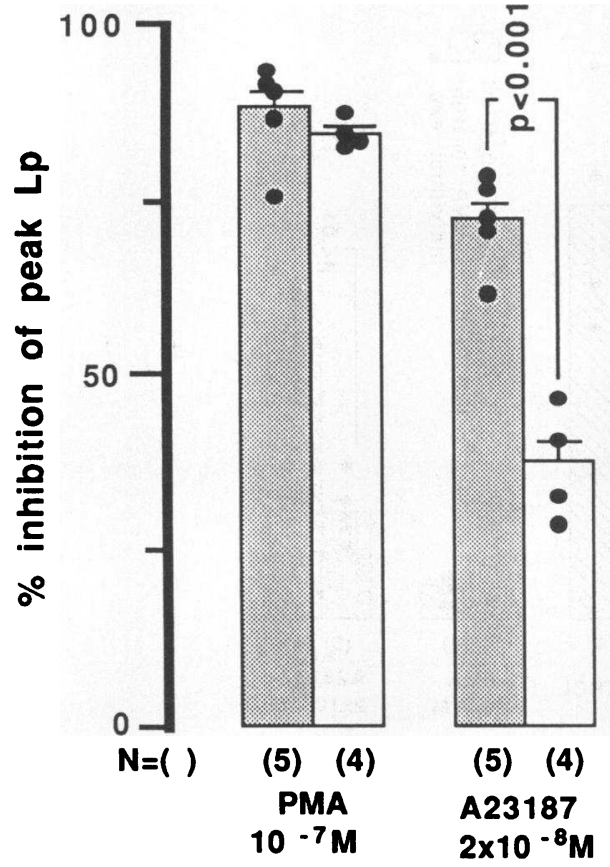

Figure 6. Effects of low $\mathrm{Ca}\left(1-2 \times 10^{-6} \mathrm{M}\right)$ bath medium on the suppressions of peak Lp by PMA or A23187. Percent inhibition of peak Lp was calculated as: [mean control peak Lp in normal or low $\mathrm{Ca}$ bath - individual experimental peak Lp in normal or low Ca bath]/ mean control peak Lp in normal or low Ca bath $\times 100 \%$. Bars above each column represent SE. N, Number of experiments. Lowering peritubular $\mathrm{Ca}$ concentration had no effect on the degree of the peak Lp suppression by PMA, but significantly reduced the degree of A23187 suppression. (ㅁ) AV, $10 \mu \mathrm{U} / \mathrm{ml}$. (ㅁ) Low Ca bath plus AVP, $10 \mu \mathrm{U} / \mathrm{ml}$. (๑) Individual experiment.

electing cellular response. In some cells, there is no interaction between these signal transduction pathways (3). In the mammalian collecting tubule, investigation of the specific interaction between these signalling pathways is a subject of great interest, since the CAMP-mediated pathway is utilized by AVP in regulating water transport of this nephron segment. We have recently demonstrated that PKC activators markedly suppress AVP-induced water transport in rabbit CCT primarily at a step distal to cAMP generation (5). Furthermore, additional evidence suggesting the physiological importance of PKC as a negative regulator of water transport has been obtained. Carbachol, a cholinergic agent, is known to stimulate PIP $_{2}$ breakdown (17) and has been found in toad bladder to inhibit AVP-induced water flow at a step distal to cAMP generation (18). Epidermal growth factor, which stimulates PIP $_{2}$ breakdown in certain cells (19), was found in our laboratory to inhibit both AVP and cAMP action on rabbit CCT water transport in a manner similar to that of PMA (20). In preliminary studies Raymond (21) showed that chronic hypokalemia suppressed the hydroosmotic effect of AVP on medullary collecting tubule, and this inhibition could be reversed by $\mathrm{H}-7$, a PKC inhibitor.

Conversely, there have been conflicting findings about the role of intracellular $\mathrm{Ca}$, another branch of $\mathrm{PIP}_{2}$ breakdown cascade, on the action of AVP. Goldfarb (6) found that increasing bath $\mathrm{Ca}$ concentration from 1.25 to $3.75 \mathrm{mM}$ augmented the hydroosmotic effect of AVP in rabbit CCT perfused in vitro at $37^{\circ} \mathrm{C}$. Dillingham et al. (8) found that $\mathrm{A} 23187$ stimulated AVP-induced water transport in rabbit CCT, and lowering bath $\mathrm{Ca}$ had an opposite effect. However, and very importantly, these experiments were conducted at $25^{\circ} \mathrm{C}$. In contrast to these stimulatory results, studies conducted at $37^{\circ} \mathrm{C}$ using agents that increase $\left[\mathrm{Ca}^{++}\right]_{\mathrm{i}}$ suggest that increased $\left[\mathrm{Ca}^{++}\right]_{\mathrm{i}}$ suppresses AVP action $(9,22)$. In amphibian urinary bladder, maneuvers that increase $\left[\mathrm{Ca}^{++}\right]_{i}$ generally suppress AVP action (18, 23-26), although Hardy (23) demonstrated that A23187 administered simultaneously with AVP augmented AVP-induced water flow. In all previous studies (18, 23-25) the concentrations of A23187 used were micromolar or greater. Such high concentrations of A23187 could produce diverse effects not specific to activation of the Ca-mediated pathway. For example, at micromolar concentrations, A23187 acts as an uncoupler of mitochondrial respiration and oxidative phosphorylation (11). In platelets, while $2-3 \times 10^{-7} \mathrm{M}$ A23187 exclusively activates the Ca-mediated pathway, doses higher than $5 \times 10^{-7} \mathrm{M}$ stimulate PKC as well (12). Both of these nonspecific actions could suppress a post-cAMP step of the AVP response $(5,27)$. Therefore it is important to use A23187 at lower than $5 \times 10^{-7} \mathrm{M}$ to examine more specifically the role of the Ca-mediated pathway (15). As $\mathrm{PIP}_{2}$ breakdown results in activation of both the PKC and the Ca-mediated pathway, it is also necessary to explore the combined effect of both pathways on AVP action to evaluate the significance of stimulated $\mathrm{PIP}_{2}$ breakdown in water transport regulation.

Our experiments performed at $37^{\circ} \mathrm{C}$ show that $\mathrm{A} 23187$ is a potent inhibitor of AVP-induced osmotic water flow when used at a concentration of $2 \times 10^{-8} \mathrm{M}$ (Fig. 3). The mechanism responsible for the opposite action of $\mathrm{A} 23187$ at $25^{\circ} \mathrm{C}$ (Fig. 2) is unknown. The hydroosmotic response of CCT to AVP is different at $25^{\circ}$ and at $37^{\circ} \mathrm{C}(28)$. The peak $\mathrm{Lp}$ at $25^{\circ} \mathrm{C}$ is lower and more stable than that at $37^{\circ} \mathrm{C}(28,29)$. Cholera toxin, an activator of stimulatory guanine nucleotide regulatory protein (Gs), which stimulates water transport by activating adenylate cyclase at $37^{\circ} \mathrm{C}$, has no effect at $25^{\circ} \mathrm{C}(30,31)$. It is also known that $\left[\mathrm{Ca}^{++}\right]_{\mathrm{i}}$ itself and $\mathrm{Ca}$-dependent calmodulin exert triphasic effects on adenylate cyclase activity: they are stimulatory at a certain $\mathrm{Ca}$ concentration and suppressive at higher or lower $\mathrm{Ca}$ concentrations (32). Therefore it is possible that increased $\left[\mathrm{Ca}^{++}\right]_{\mathrm{i}}$ has different effects on AVP-induced water transport at different temperatures.

There are some similarities between the actions of PMA and A23187: both increased basal Lp slightly and were equally potent inhibitors of AVP response either in pre- or posttreatment studies (5). Both agents also have been found to inhibit $\mathrm{Na}$ transport in this nephron segment $(33,34)$. Furthermore, the suppressive effects of these agents are additive (Fig. 3). Thus it is important to examine whether the PKC pathway and the Ca-mediated pathway act through a common mechanism or not.

Our series of studies clearly demonstrate the difference between these two pathways in their suppression of the AVP action. As shown in Fig. 2, A23187 suppressed the AVP action predominantly at a pre-cAMP step. This is in accord with the inhibitory effect of $\mathrm{Ca}$ on cAMP generation or accumulation reported by other investigators (35-37). Jones et al. (9) also found that pre-cAMP factors, i.e., stimulation of prostaglandin synthesis and phosphodiesterase, mainly mediated the suppression of AVP-induced Lp by Ca. In our experiments, A23187 partially (38\%) suppressed the CcAMP-induced peak Lp only at a micromolar concentration $\left(2 \times 10^{-6} \mathrm{M}\right)$. This 
finding is essentially the same as recent studies by Taylor et al. (25) or Lorenzen et al. (22). They found that increasing $\left[\mathrm{Ca}^{++}\right]_{\mathrm{i}}$ with micromolar A23187 or quinidine partially inhibit cAMP-induced water transport. In their studies, as well as our micromolar A23187 study, the Ca inhibition of cAMP action was smaller than that of AVP action. This suggests that the $\mathrm{Ca}$ action is both pre- and post-cAMP when high concentration of A23187 or quinidine were used. However, we can not exclude the possibility that nonspecific or toxic effects mentioned above are seriously involved in these post-cAMP suppression. The suppression by $2 \times 10^{-8} \mathrm{M}$ A23187 was not reversed by SSP (Fig. 4). This clarified that the action of A23187 at this concentration is not mediated by activation of PKC, which can be caused by micromolar A23187 (15). The action of 2 $\times 10^{-8} \mathrm{M} \mathrm{A} 23187$ was significantly attenuated by removal of bath $\mathrm{Ca}$ (Fig. 6). Failure of complete reversal might be due to a small increase in $\left[\mathrm{Ca}^{++}\right]_{i}$ by influx of the remaining peritubular $\mathrm{Ca}$, or by $\mathrm{Ca}$ release from intracellular storage $(4,38)$. At any rate this attenuation means that a $\mathrm{Ca}$ ionophoric action is responsible for the A23187 inhibition. Our present studies also demonstrate that the A23187 action is largely dependent on IND-inhibitable cyclooxygenase metabolites of arachidonate (Fig. 5). This is in agreement with previous studies in CCT (9) or toad bladders $(24,39)$. Incomplete reversal by IND could be explained by the existance of other mechanisms of the $\mathrm{Ca}$ inhibition. Stimulation of cAMP phosphodiesterase by $\mathrm{Ca}$ is well known (35), and its involvement in the Ca-mediated inhibition of AVP-induced water transport has been confirmed (9). Calcium also stimulates arachidonic acid release by activating phospholipase $A_{2}(40)$. Thus it is also possible that increased synthesis of noncyclooxygenase metabolites of arachidonate are responsible for the $\mathrm{Ca}$ inhibition. Indeed, in our laboratory, certain epoxygenase metabolites of arachidonate have been found to inhibit AVP-induced water transport (41).

Conversely, PMA (either at $10^{-7}$ or $10^{-9} \mathrm{M}$ ) suppression of AVP action is primarily at a post-cAMP step as we have previously demonstrated (5). The peak Lp suppression by $10^{-7} \mathrm{M}$ PMA was totally abolished by inhibition of PKC with SSP (Fig. 4). This suggests that the action of PMA is exclusively via activation of PKC. IND pretreatment could not reverse the suppression by either $10^{-7}$ or $10^{-9}$ M PMA (Fig. 5). Accordingly, the PMA action on AVP-induced water transport can not be mediated by stimulation of prostaglandin synthesis, which has been observed in Madin Darby canine kidney (MDCK) cells in response to PKC activation (42). Increased $\mathrm{Ca}$ influx is known to result from PKC activation in some cell types $(43,44)$. However, it would not be responsible for the suppression of AVP action, since lowering bath $\mathrm{Ca}$ concentration failed to attenuate the peak Lp suppression by $10^{-7} \mathrm{M}$ PMA, while it significantly attenuated the A23187 suppression (Fig. 6). We did not examine the effect of low Ca bath on the suppression by $10^{-9}$ M PMA. If this concentration of PMA exerts the suppression, even in part, via increasing $\mathrm{Ca}$ influx like A23187, IND must attenuate this submaximal PMA action; but it did not.

In summary, our studies suggest (a) Both branches of the $\mathrm{PIP}_{2}$ hydrolysis cascade are potent negative modulators of the hydroosmotic effect of AVP in rabbit CCT; $(b)$ Activation of PKC and elevation of $\left[\mathrm{Ca}^{++}\right]_{i}$ are additive in their suppression of AVP action; (c) The Ca-mediated pathway preferentially blocks a pre-cAMP step, and this inhibition is partly mediated by cyclooxygenase metabolites, but not by PKC activation; and $(d)$ The PKC pathway predominantly blocks a post-cAMP step, and this action is independent of either peritubular $\mathrm{Ca}$ or cyclooxigenase metabolites.

\section{Acknowledgments}

The authors wish to thank Ms. Deborah Asbell-Gillespie for her skillful technical assistance.

This study was supported by National Institutes of Health grant AM-37097 and Veterans Administration grant 151JAI-733.

\section{References}

1. Exton, J. H. 1985. Role of calcium and phosphoinositides in the action of certain hormones and neurotransmitters. J. Clin. Invest. 75:1753-1757.

2. Mitchell, R. H. 1983. Polyphosphoinositide breakdown as the initiating reaction in receptor stimulated inositol phospholipid metabolism. Life Sci. 32:2083-2085.

3. Takai, Y., U. Kikkawa, K. Kaibuchi, and Y. Nishizuka. 1984. Membrane phospholipid metabolism and signal transduction for protein phosphorylation. In Advance in Cyclic Nucleotide and Protein Phosphorylation Research. Vol. 18. P. Greengard and G. A. Robinson, editors. Raven Press, New York. 119-158.

4. Rasmussen, H., I. Kojima, K. Kojima, W. Zawalich, and W. Apfeldort. 1984. Calcium as intracellular messenger: sensitivity modulation, C-kinase pathway, and sustained cellular response. In Advance in Cyclic Nucleotide and Protein Phosphorylation Research. Vol. 18. P. Greengard and G. A. Robinson, editors. Raven Press, New York. 159-193.

5. Ando, Y., H. R. Jacobson, and M. B. Breyer. 1987. Phorbol myristate acetate, dioctanoylglycerol, and phosphatidic acid inhibit the hydroosmotic effect of vasopressin on rabbit cortical collecting tubule. J. Clin. Invest. 80:590-593.

6. Goldbarb, S. 1982. Effects of calcium on ADH action in the cortical collecting tubule perfused in vitro. Am. J. Physiol. 243(Renal Fluid Electrolyte Physiol. 12):F481-F486.

7. Jones, S. M., G. Frindt, and E. E. Windhager. 1985. Peritubular $\mathrm{Ca}$ and the hydroosmotic response to $\mathrm{ADH}$ in rabbit cortical collecting tubules (CCT). Fed. Proc. 44:1015. (Abstr.)

8. Dillingham, M. A., B. S. Dixon, and R. J. Anderson. 1987. Calcium modulates vasopressin effect in rabbit cortical collecting tubule. Am. J. Physiol. 252(Renal Fluid Electrolyte Physiol. 21):F115F121.

9. Jones, S. M., G. Frindt, and E. E. Windhager. 1987. Mechanism of inhibition of hydroosmotic response to vasopressin (VP) by ionomycin in rabbit cortical collecting tubule (CCT). Fed. Proc. 46:1284. (Abstr.)

10. DuBois, R., A. Verniory, and M. Abramow. 1976. Computation of the osmotic water permeability of perfused tubule segments. Kidney Int. 10:478-479.

11. Humes, H. D., and J. M. Weinberg. 1980. Mechanism of calcium ionophore A23187 (I) induced declines in active ion transport across transporting epithelia. Clin. Res. 28:449A. (Abstr.)

12. Kaibuchi, K., Y. Takai, M. Sawamura, M. Hoshijima, T. Fujikura, and Y. Nishizuka. 1983. Synergistic functions of protein phosphorylation and calcium mobilization in platelet activation. J. Biol. Chem. 258:6701-6704.

13. Erne, P., M. Schachter, D. Fabbro, C. M. M. Miles, and P. S. Sever. 1987. Calcium transient in human platelets monitored by aequorin, fura-2, and quin-2: effects of protein kinase $C$ activation and inhibition. Biochem. Biophys. Res. Commun. 145:66-72.

14. Flower, R. J. 1974. Drugs which inhibit prostaglandin synthesis in various tissues or cells. Pharmacol. Rev. 26:33-67.

15. Nishizuka, Y. 1984. The role of protein kinase $C$ in cell surface signal transduction and tumor promotion. Nature (Lond.). 308:693698. 
16. Kikkawa, U., and Y. Nishizuka. 1986. The role of protein kinase $\mathrm{C}$ in transmembrane signaling. Ann. Rev. Cell Biol. 2:149-178.

17. Takuwa, Y., N. Takuwa, and H. Rasmussen. 1987. Carbacol induces a rapid and sustained hydrolysis of polyphosphoinositide in bovine tracheal smooth muscle measurements of the mass of polyphosphoinisitides, 1.2-diacylglycerol, and phosphatidic acid. J. Biol. Chem. 261:14670-14675.

18. Arruda, J. A. L., and S. Sabatini. 1980. Cholinergic modulation of water transport in the toad bladder. Am. J. Physiol. 239(Renal Fluid Electrolyte Physiol. 8):F154-F159.

19. Saywer, S. T., and S. Cohen. 1981. Enhancement of calcium uptake and phosphatidylinositol turnover by epidermal growth factor in A-431 cells. Biochemistry. 20:6280-6286.

20. Breyer, M. D., H. R. Jacobson, and J. Breyer. Epidermal growth factor (EGF) inhibits both chlorophenylthio-cyclic AMP (CcAMP) and vasopressin (VP) stimulated hydraulic conductivity (Lp) in the rabbit cortical collecting tubule (CCT). Kidney Int. 33:255. (Abstr.)

21. Raymond, K. H. 1987. In vitro effect of protein kinase C (PKC) inhibition on AVP stimulated hydraulic conductivity coefficient (Lp) in outer medullary collecting tubules (OOMCT) from control (C) and potassium depleted (KD) rabbits. Clin. Res. 35:555A. (Abstr.)

22. Lorenzen, M., G. Frindt, A. Taylor, and E. E. Windhager 1987. Quinidine effect on hydrosmotic response of collecting tubules to vasopressin and cAMP. Am. J. Physiol. 134(Renal Fluid and Electrolyte Physiol. 21):F1103-F1111.

23. Hardy, M. A. 1978. Intracellular calcium as a modulator of transepithelial permeability to water in frog urinary bladder. J. Cell Biol. 76:787-791.

24. Yorio, T., S. L. Henry, D. H. Hodges, and J. L. Caffrey. 1983. Role of calcium and prostaglandins in the antidiuretic hormone response: effect of ionophore A23187. Biochem. Pharmacol. 32:11131118

25. Taylor, A., E. Eich, M. Pearl, A. S. Brem, and E. Q. Peeper. 1987. Cytosolic calcium and the action of vasopressin in toad urinary bladder. Am. J. Physiol. 252(Renal Fluid Electrolyte Physiol. 21):F1028-F1041.

26. Sabatini, S. 1986. Parathyroid hormone inhibits water flow in the isolated toad bladder. Am. J. Physiol. 250(Renal Fluid Electrolyte Physiol. 19):F532-538.

27. Handler, J., M. Peterson, and J. Orloff. 1966. Effect of metabolic inhibitors on the response of toad bladder to vasopressin. Am. J. Physiol. 211:1175-1180.

28. Hall, D., and J. J. Grantham. 1980. Temperature effect on ADH response of isolated perfused rabbit collecting tubules. Am. J. Physiol. 239(Renal Fluid Electrolyte Physiol. 8):F595-F601.

29. Cogan, E., and M. Abramow. 1986. Inhibition by lithium of hydroosmotic action of vasopressin in the isolated perfused cortical collecting tubule of the rabbit. J. Clin. Invest. 77:1507-1514.

30. Nadler, S. P., S. C. Hebert, and B. M. Brenner. 1986. PGE ${ }_{2}$, forskolin, and cholera toxin interactions in rabbit cortical collecting tubule. Am. J. Physiol. 250(Renal Fluid Electrolyte Physiol. 19):F127-F135.

31. Hoch, B. S., and S. D. Levine. 1987. Cholera toxin enhances adenylate cyclase dependent transport in toad urinary bladder. Am. J. Physiol. 252(Renal Fluid Electrolyte Physiol. 21):F621-F626.

32. Ausiello, D. A., and D. Hall. 1981. Regulation of vasopressin sensitive adenylate cyclase by calmodulin. J. Biol. Chem. 256:97969798.

33. Hays, S. R., M. Baum, and J. P. Kokko. 1987. Phorbol esters inhibit sodium absorption and $\mathrm{K}$ secretion in the rabbit cortical collecting tubule (CCT). Kidney Int. 31:435. (Abstr.)

34. Palmer, L. G., and G. Frindt. 1984. Ca ionophore and phorbol ester inhibit Na channels in rat cortical collecting tubules. Fed. Proc. 46:495. (Abstr.)

35. Kusano, E., N. Murayama, J. L. Werness, S. Christensen, S. Homma, A. N. K. Yusufi, and T. P. Dousa. 1985. Effects of calcium on the vasopressin-sensitive cAMP metabolism in medullary tubules. Am. J. Physiol. 249(Renal Fluid Electrolyte Physiol. 18):F956-F966.

36. Marumo, F., and I. S. Edelman. 1971. Effects of $\mathrm{Ca}^{++}$and prostaglandin $E_{1}$ on vasopressin activation of renal adenyl cyclase. $J$. Clin. Invest. 50:1613-1620.

37. Argy, W. P., J. S. Handler, and J. Orloff. 1967. $\mathrm{Ca}^{++}$and $\mathrm{Mg}^{++}$ effects on toad bladder response to cyclic AMP, theophylline, and ADH analogues. Am. J. Physiol. 213:803-808.

38. Moolenaar, W. H., W. Kruijer, B. C. Tilly, I. Verlaan, A. J. Bierman, and S. W. de Laat. 1986. Growth factor-like action of phosphatidic acid. Nature (Lond.). 323:171-173.

39. Arruda, J. A. L. 1982. Interaction of calcium and cyclooxygenase inhibitors on transport by the turtle and toad bladder. Arch. Int. Pharmacodyn. 257:319-334.

40. Craven, P. A., and F. R. DeRubertis. 1983. $\mathrm{Ca}^{2+}$ Calmodulindependent release of arachidonoc acid for renal medullary prostaglandin synthesis. J. Biol. Chem. 258:4814-4823.

41. Hirt, D., J. Falck, J. Capdevila, M. Breyer, and H. Jacobson. 1987. 5,6 dihydroxyeicosatrienoic acid modulates the hydrosmotic effect of vasopressin on rabbit cortical collecting tubule. Clin. Res. 35:635A. (Abstr.)

42. Ouchi, K., and L. Levine. 1978. Stimulation of prostaglandin synthesis by tumor promoting phorbol-12,13-diesters in canine kidney (MDCK) cells. J. Biol. Chem. 253:4783-4790.

43. De Reimer, S. A., J. A. Strong, K. A. Albert, P. Greengard, and L. K. Kaczmarek. 1984. Enhancement of calcium current in Aplysia neurones by phorbol ester and protein kinase C. Nature (Lond.). 313:313-315.

44. Kurtz, A., J. Pfeilschifter, A. Hutter, C. Buerle, R. Nobling, R. Traugner, E. Hackenthal, and C. Bauer. 1986. Role of protein kinase C in inhibition of renin release caused by vasoconstrictors. Am. J. Physiol. 250(Cell Physiol. 19):C563-571. 